\title{
Indispensable tools: embryonic stem cells yield insights into the human heart
}

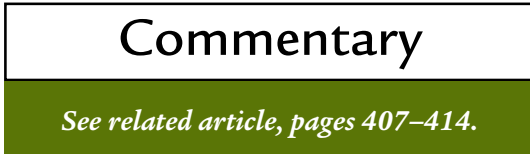

\author{
J. Hescheler and B.K. Fleischmann \\ Institute of Neurophysiology, University of Cologne, Cologne, Germany
}

Address correspondence to: J. Hescheler, Institute of Neurophysiology, University of Cologne, Robert-Kochstrasse 39, D-50931 Koeln, Germany. Phone: 49-221-478-6960; Fax: 49-221-478-3834; E-mail: j.hescheler@uni-koeln.de.

J. Clin. Invest. 108:363-364 (2001). DOI:10.1172/JCI200113634.

This Commentary is written by rather disappointed German scientists who are not allowed to establish human embryonic stem cells and have been publicly blamed for using them. While Germany is in a state of turmoil over sensitive questions regarding the possibility of importing of human stem cell lines for research purposes, other countries, Israel among them, are actively supporting basic research on this interesting topic.

In the current issue of the JCI, the fruits of this liberal approach toward basic science are shown by Kehat and coworkers from the Technion-Israel Institute of Technology in Haifa (1). The authors clearly demonstrate that the human embryonic stem cell line H9.2, first reported by Thomson et al. (2), can be differentiated into cardiomyocytes. These data go beyond the previously reported evidence that the human embryonic stem cells can

This study provides the first compelling evidence that human embryonic stem cells can be differentiated into cardiomyocytes.

differentiate into the ectodermal, endodermal, and mesodermal lineages (3). Although substantial additional information is necessary, particularly regarding the functional properties of these cells, the authors show, on the basis of gene expression, ultrastructure, immunofluorescence, and basic functional tests, that the derived cells have the properties of cardiomyocytes. Thus, this study provides the first compelling evidence that human embryonic stem cells can be differentiated into cardiomyocytes, a notion that has been well established for the murine embryonic stem cell system (refs. 4-6; for review see ref. 7). These previous studies, documenting the morphological as well as the functional integrity of murine embryonic stem cell-derived cardiomyocytes, should now be experimentally transferred to the human cells in order to further corroborate their physiological integrity.

The present paper (1) also brings to light certain novel, apparently speciesdependent features of embryonic development. Whereas the murine gestation period lasts for 20 days, human gestation takes approximately 13 times longer, a difference that may contribute to the significantly longer time needed for human cardiomyocyte differentiation in culture. However, as the authors point out, culture conditions, the use of other types of feeder cells, and growth factors may in the future result in a better synchronization of the initiation of spontaneous beating

may improve the efficiency of cardiomyocyte generation. From a scientific point of view, it will be fascinating to compare molecular, as well as functional, aspects of early stages of development in human embryonic stem cells and in the murine system.

The most interesting aspect of the present article (1), in our view, is the possibility of using human embryonic stem cells as a source for cell replacement or growing organ tissue, such as structures in vitro for transplantation purposes. Similarly, we (8) and others (9-11) have shown that early embryonic cardiomyocytes, including those derived from murine embryonic stem cells (12), are well suited for cellular replacement therapy after heart injury. The repaired heart performs better and, most importantly, improves the survival rate of the mice upon cryoinjury (8).

Experiments to test the potential of human embryonic stem cell-derived cardiomyocytes will be critical for developing their therapeutic potential. Also crucial will be a better understanding of key signal transduction pathways and cellular factors activated in the lesioned heart and of their effects on transplanted cells. Furthermore, convincing functional measurements are needed to show the coupling of transplanted cardiomyocytes with the surrounding native heart tissue.

We are convinced that the successful differentiation of human embryonic stem cells into cardiomyocytes is an important step toward understanding the early process of heart development and analyzing their potential for cellular replacement therapies. Furthermore, it provides scientists with a formidable tool to compare the therapeutic efficiency of embryonic versus adult stem cells. It may also prove very helpful for the testing of pharmacological agents onto cardiomyocytes in vitro. Due to the enormous potential of human embryonic stem cells for basic and applied research, efforts should be made to promote publicly funded research and to prevent domination by industrial research.

Science appears to have reached a point of decision. Experts throughout the world are in agreement that research on human stem cells could bring an enormous step forward in the field of medicine. In the 14th century, the introduction of human dissection for anatomical purposes was indeed a matter of great dispute. At that time 
many offered great resistance on ethical grounds to such innovation. It was only due to a few physicians who had the courage to override such resistance that a much deeper knowledge of medicine was achieved - from which we continue to reap benefits even today. The decision makers of today should not block the future path of basic and clinical research.

1. Kehat, I., et al. 2001. Human embryonic stem cells can differentiate into myocytes with structural and functional properties of cardiomyocytes. J. Clin. Invest. 108:407-414.

2. Thomson, J.A., et al. 1998. Embryonic stem cell lines derived from human blastocysts. Science. 282:1145-1147.

3. Schuldiner, M., Yanuka, O., Itskovitz-Eldor, J.,
Melton, D.A., and Benvenisty, N. 2000. Effects of eight growth factors on the differentiation of cells derived from human embryonic stem cells. Proc. Natl. Acad. Sci. USA. 97:11307-11312.

4. Wobus, A.M., Wallukat, G., and Hescheler, J. 1991. Pluripotent mouse embryonic stem cells are able to differentiate into cardiomyocytes expressing chronotropic responses to adrenergic and cholinergic agents and $\mathrm{Ca} 2+$ channel blockers. Differentiation. 48:173-182.

5. Maltsev, V.A., Wobus, A.M., Rohwedel, J., Bader, M., and Hescheler, J. 1994. Cardiomyocytes dif ferentiated in vitro from embryonic stem cells developmentally express cardiac-specific genes and ionic currents. Circ. Res. 75:233-244.

6. Hescheler, J., et al. 1997. Embryonic stem cells: a model to study structural and functional properties in cardiomyogenesis. Cardiovasc. Res. 36:149-162.

7. Hescheler, J., et al. 1999. Establishment of ionic channels and signalling cascades in the embryonic stem cell-derived primitive endoderm and cardiovascular system. Cells Tissues Organs. 165:153-164.

8. Roell, W., et al. 2001. Transplantation of transgenic embryonic cardiomyocytes in a mouse model. Pflugers Arch. 441:153. (Abstr.)

9. Koh, G.Y., et al. 1995. Stable fetal cardiomyocyte grafts in the hearts of dystrophic mice and dogs. J. Clin. Invest. 96:2034-2042.

10. Li, R.K., et al. 1997. Natural history of fetal rat cardiomyocytes transplanted into adult rat myocardial scar tissue. Circulation. 96(Suppl.):II176-II-186.

11. Reinecke, H., Zhang, M., Bartosek, T., and Murry, C.E. 1999. Survival, integration and differentiation of cardiomyocytes grafts: a study in normal and injured rat hearts. Circulation. 100:193-202.

12. Klug, M.G., Soonpaa, M.H., Koh, G.Y., and Field, L.J. 1996. Genetically selected cardiomyocytes from differentiating embronic stem cells form stable intracardiac grafts. J. Clin. Invest. 98:216-224. 